\title{
PI control of discrete linear repetitive processes ${ }^{\text {tr }}$
}

\author{
Bartlomiej Sulikowski ${ }^{a}$, Krzysztof Galkowski ${ }^{a}$, Eric Rogers ${ }^{\mathrm{b}, *}$, David H. Owens ${ }^{\mathrm{c}}$ \\ ${ }^{a}$ Institute of Control and Computation Engineering, University of Zielona Gora, ul. Podgorna 50, 65-246 Zielona Gora, Poland \\ ${ }^{\mathrm{b}}$ School of Electronics and Computer Science, University of Southampton, Southampton, SO17 1BJ, UK \\ ${ }^{\mathrm{c}}$ Department of Automatic Control and Systems Engineering, University of Sheffield, Sheffield S1 3JD, UK
}

Received 8 March 2005; received in revised form 21 December 2005; accepted 17 January 2006

Available online 10 March 2006

\begin{abstract}
Repetitive processes are a distinct class of 2D systems (i.e. information propagation in two independent directions) of both systems theoretic and applications interest. They cannot be controlled by direct extension of existing techniques from either standard (termed 1D here) or 2D systems theory. In this paper, we exploit their unique physical structure to show how two term, i.e. proportional plus integral (or PI) action, can be used to control these processes to produce desired behavior (as opposed to just stability).
\end{abstract}

(C) 2006 Elsevier Ltd. All rights reserved.

Keywords: Repetitive dynamics; Stability; Stabilization; Controller design; LMI

\section{Introduction}

The essential unique characteristic of a repetitive process is a series of sweeps, termed passes, through a set of dynamics defined over a fixed finite duration known as the pass length. On each pass an output, termed the pass profile, is produced which acts as a forcing function on, and hence contributes to, the dynamics of the next pass profile. This, in turn, leads to the unique control problem for these processes in that the output sequence of pass profiles generated can contain oscillations that increase in amplitude in the pass-to-pass direction.

Physical examples of repetitive processes include long-wall coal cutting and metal rolling operations (see, for example, Rogers \& Owens, 1992). Also in recent years applications have arisen where adopting a repetitive process setting for analysis has distinct advantages over alternatives. They can, for example, be used as an analysis tool in iterative learning control (ILC)

\footnotetext{
This paper was not presented at any IFAC meeting. This paper was recommended for publication in revised form by Associate Editor Karl Henrik Johansson under the direction of Editor Andre' Tits.

* Corresponding author. Tel.: +44 1703592197; fax: +44 1703594498.

E-mail addresses: b.sulikowski@issi.uz.zgora.pl (B. Sulikowski), k.galkowski@issi.uz.zgora.pl (K. Galkowski), etar@ecs.soton.ac.uk (E. Rogers), D.H.Owens@sheffield.ac.uk (D.H. Owens).
}

schemes as fully explained in Owens, Amann, Rogers, and French (2000). In this paper we consider control in the presence of disturbances for so-called discrete linear repetitive processes.

Throughout this paper, the null matrix and the identity matrix with the required dimensions are denoted by 0 and $I$, respectively. Moreover, $M>0(<0)$ denotes a real symmetric positive (negative) definite matrix.

\section{Background}

Following (Rogers \& Owens, 1992) the state space model of a discrete linear repetitive process has the following form over $0 \leqslant p \leqslant \alpha-1, k \geqslant 0$ :

$$
\begin{aligned}
& x_{k+1}(p+1)=A x_{k+1}(p)+B u_{k+1}(p)+B_{0} y_{k}(p)+E w(p), \\
& y_{k+1}(p)=C x_{k+1}(p)+D u_{k+1}(p)+D_{0} y_{k}(p)+F w(p) .
\end{aligned}
$$

Here on pass $k, x_{k}(p) \in \mathbb{R}^{n}$ is the state vector, $y_{k}(p) \in \mathbb{R}^{m}$ is the pass profile vector, $u_{k}(p) \in \mathbb{R}^{r}$ is the input vector and $w(p) \in \mathbb{R}^{q}$ a disturbance vector and here is assumed to be the same on each pass. Also the boundary conditions are, without loss of generality, taken as zero initial pass profile and $x_{k+1}(0)=$ $d_{k+1}, k \geqslant 0$, where $d_{k+1}$ has constant entries. 
This state space model allows for disturbances which enter both the state and pass profile dynamics on each pass. The stability theory (Rogers \& Owens, 1992) for linear repetitive processes is based on an abstract model in a Banach space setting which includes the vast majority of such processes as special cases. In this model it is the pass-to-pass coupling (noting again the unique control problem for them) which is critical. This is of the form $y_{k+1}=L_{\alpha} y_{k}$, where $y_{k} \in E_{\alpha}\left(E_{\alpha}\right.$ a Banach space with norm $\|\cdot\|)$ and $L_{\alpha}$ is a bounded linear operator mapping $E_{\alpha}$ into itself. Two concepts of stability can be defined but in general it is the stronger of these, so-called stability along the pass which is required. This holds if, and only if there exist numbers $M_{\infty}>0$ and $\lambda_{\infty} \in(0,1)$ independent of $\alpha$ such that $\left\|L_{\alpha}^{k}\right\| \leqslant M_{\infty} \lambda_{\infty}^{k}, k \geqslant 0$ (where $\|\cdot\|$ also denotes the induced operator norm) and can be interpreted as bounded-input boundedoutput stability independent of the pass length.

It can be shown from results in Rogers and Owens (1992) that stability along the pass holds for (1) if, and only if,

$\mathscr{C}\left(\mathrm{z}_{1}, \mathrm{z}_{2}\right)=\operatorname{det}\left(I-\mathrm{z}_{1} \widehat{A}_{1}-\mathrm{z}_{2} \widehat{A}_{2}\right) \neq 0$ in $\bar{U}^{2}$,

where $\bar{U}^{2}=\left\{\left(\mathrm{z}_{1}, \mathrm{z}_{2}\right):\left|\mathrm{z}_{1}\right| \leqslant 1,\left|\mathrm{z}_{2}\right| \leqslant 1\right\}$, and

$\widehat{A}_{1}=\left[\begin{array}{cc}A & B_{0} \\ 0 & 0\end{array}\right], \quad \widehat{A}_{2}=\left[\begin{array}{cc}0 & 0 \\ C & D_{0}\end{array}\right]$.

In this work, we use the following LMI based sufficient condition derived from (2) which, unlike all other existing stability tests, leads immediately (see below) to systematic methods for control law design.

Theorem 1 (Gatkowski et al., 2003). A discrete linear repetitive process described by (1) is stable along the pass if there exist matrices $Y>0$ and $Z>0$ such that the following $L M I$ holds:

$$
\left[\begin{array}{ccc}
Z-Y & 0 & Y \widehat{A}_{1}^{\mathrm{T}} \\
0 & -Z & Y \widehat{A}_{2}^{\mathrm{T}} \\
\widehat{A}_{1} Y & \widehat{A}_{2} Y & -Y
\end{array}\right]<0 .
$$

It is also possible to represent discrete linear repetitive processes of the form considered here in terms of the models arising in the analysis and control of spatially distributed systems, e.g., Dullerud and D'Andrea (2004). In fact, it is a Roesser model interpretation of the process dynamics which results, whose role in onward analysis has already been extensively investigated, e.g. Rogers and Owens (1992). Moreover, such a model removes the key unique feature of these processes, i.e. repeated sweeps through a set of dynamics defined over the finite pass length with resetting of part of the boundary conditions (the pass state initial vector) before the start of each new pass. Note also that the boundary conditions can be an explicit function of the previous pass profile and this alone can cause instability. Clearly, however, proper exploitation of the links (at the level of the model structures and the analysis tools/control law design algorithms) between these two areas could be highly beneficial (in both directions).

\section{PI control}

In terms of "acceptable", or desired, performance from a given example, it is clear that stability along the pass must hold and how to ensure this property has been the subject of recent research (Gałkowski et al., 2003). In particular, it has been shown (by exploiting Theorem 1) that a control law of the form

$u_{k+1}(p)=K_{x} x_{k+1}(p)+K_{z 1} y_{k}(p)$

can be used to give this property where $K_{x}$ and $K_{z 1}$ are compatibly dimensioned matrices to be designed.

If stability along the pass holds, then the resulting pass profile sequence converges strongly, i.e. in the sense of the norm on $E_{\alpha}$ in the pass-to-pass direction to a so-called steady or limit profile denoted by $y_{\infty}$. Also for the processes considered here, $y_{\infty}$ is described by a stable 1D discrete linear systems statespace model. The new question solved in this paper is how to obtain a specified limit profile $y_{\text {ref }}(p)$ even in the presence of disturbance terms, where the transient characteristics of $y_{\infty}$ are most naturally defined in terms of standard 1D measures.

Consider (1) at point $p$ on pass $k$. Then the total tracking error at this point is defined as

$\chi_{k}(p):=\sum_{j=0}^{k}\left(y_{j}(p)-y_{\text {ref }}(p)\right)$

i.e. the error at point $p$ summed from pass 0 to $k$. Now, introduce the so-called extended output vector $z_{k+1}(p):=$ $\left[y_{k+1}^{\mathrm{T}}(p) \chi_{k+1}^{\mathrm{T}}(p)\right]^{\mathrm{T}}$. Then substitution from the process statespace model and some routine re-arranging yields

$$
\begin{aligned}
z_{k+1}(p)= & {\left[\begin{array}{l}
C \\
C
\end{array}\right] x_{k+1}(p)+\left[\begin{array}{cc}
D_{0} & 0 \\
D_{0} & I
\end{array}\right] z_{k}(p) } \\
& +\left[\begin{array}{l}
D \\
D
\end{array}\right] u_{k+1}(p)+\left[\begin{array}{c}
0 \\
-I
\end{array}\right] y_{\mathrm{ref}}(p)+\left[\begin{array}{c}
F \\
F
\end{array}\right] w(p) .
\end{aligned}
$$

Suppose now that as $k \rightarrow \infty, x_{k}(p) \rightarrow x_{\infty}(p), z_{k}(p) \rightarrow$ $z_{\infty}(p), u_{k}(p) \rightarrow u_{\infty}(p)$, and let $\chi_{\infty}(p)$ denote $\lim _{k \rightarrow \infty} \chi_{k}(p)$. Also define the following so-called incremental vectors $\hat{z}_{k}(p)=$ $z_{k}(p)-z_{\infty}(p), \hat{u}_{k}(p)=u_{k}(p)-u_{\infty}(p), \hat{x}_{k}(p)=x_{k}(p)-$ $x_{\infty}(p)$.Then using (1) and (5) we obtain

$\hat{x}_{k+1}(p+1)=A \hat{x}_{k+1}(p)+\hat{B}_{0} \hat{z}_{k}(p)+B \hat{u}_{k+1}(p)$,

$\hat{z}_{k+1}(p)=\hat{C} \hat{x}_{k+1}(p)+\hat{D}_{0} \hat{z}_{k}(p)+\hat{D} \hat{u}_{k+1}(p)$,

where

$\hat{B}_{0}=\left[\begin{array}{ll}B_{0} & 0\end{array}\right], \quad \hat{C}=\left[\begin{array}{l}C \\ C\end{array}\right], \quad \hat{D}_{0}=\left[\begin{array}{cc}D_{0} & 0 \\ D_{0} & I\end{array}\right]$,

$\hat{D}=\left[\begin{array}{l}D \\ D\end{array}\right]$

and the key point now is that the influence of the disturbance has been completely rejected. The task now is to meet the specification that the limit profile (for the original process) be equal to the prescribed vector $y_{\text {ref }}(p)$.

In (6), the matrix $\hat{D}_{0}$ always has eigenvalues with modulus at least equal to unity and therefore (from (2)) a necessary 
condition for stability along the pass does not hold. Hence to obtain any limit profile from it control action must be applied. Consequently consider applying the control law

$\hat{u}_{k+1}(p)=K_{x} \hat{x}_{k+1}(p)+K_{z 1} \hat{y}_{k}(p)+K_{z 2} \hat{\chi}_{k}(p)$

to (6). Then the following result whose proof follows immediately on interpreting Theorem 1 for the controlled process state-space model and hence the details are omitted here.

Theorem 2. Suppose that a control law of the form (7) is applied to a discrete linear repetitive process described by a state-space model of the form (6). Then the resulting process is stable along the pass if there exists matrices $Y>0, Z>0$ and $N$ such that the following LMI holds:

$\left[\begin{array}{ccc}Z-Y & 0 & Y \widetilde{A}_{1}^{\mathrm{T}}+N^{\mathrm{T}} \widetilde{B}_{1}^{\mathrm{T}} \\ 0 & -Z & Y \widetilde{A}_{2}^{\mathrm{T}}+N^{\mathrm{T}} \widetilde{B}_{2}^{\mathrm{T}} \\ \widetilde{A}_{1} Y+\widetilde{B}_{1} N & \widetilde{A}_{2} Y+\widetilde{B}_{2} N & -Y\end{array}\right]<0$

where $\widetilde{B}_{1}=\left[\begin{array}{ll}B^{\mathrm{T}} & 0\end{array}\right]^{\mathrm{T}}, \widetilde{B}_{2}=\left[\begin{array}{ll}0 & \hat{D}^{\mathrm{T}}\end{array}\right]^{\mathrm{T}}$ and

$\widetilde{A}_{1}=\left[\begin{array}{cc}A & \hat{B}_{0} \\ 0 & 0\end{array}\right], \quad \widetilde{A}_{2}=\left[\begin{array}{cc}0 & 0 \\ \hat{C} & \hat{D}_{0}\end{array}\right]$.

If this condition holds then the matrices in the control law are given by

$\left[\begin{array}{lll}K_{x} & K_{z 1} & K_{z 2}\end{array}\right]=N Y^{-1}$.

Suppose now that this last result holds. Then it follows immediately that $y_{\infty}(p)=y_{\text {ref }}(p)$ as required. Moreover,

$$
\begin{aligned}
u_{k+1}(p)= & K_{x}\left(x_{k+1}(p)-x_{\infty}(p)\right)+K_{z 1}\left(y_{k}(p)-y_{\text {ref }}(p)\right) \\
& +K_{z 2}\left(\chi_{k}(p)-\chi_{\infty}(p)\right)+u_{\infty}(p) .
\end{aligned}
$$

As the final form of the control law to be applied to the original process is

$u_{k+1}(p)=\left[\begin{array}{ll}K_{x} & K_{z 1}\end{array}\right]\left[\begin{array}{c}x_{k+1}(p) \\ y_{k}(p)\end{array}\right]+K_{z 2} \chi_{k}(p)$,

where $\left(X_{k+1}^{a}\right)(p):=\left[x_{k+1}^{\mathrm{T}}(p) y_{k}^{\mathrm{T}}(p)\right]^{\mathrm{T}}$ is termed the augmented state vector, we also have

$-K_{x} x_{\infty}(p)-K_{z 1} y_{\text {ref }}(p)-K_{z 2} \chi_{\infty}(p)+u_{\infty}(p)=0$.

Hence the final control law (8) has a two term structure, where the first term (involving $X_{k+1}^{a}(p)$ ) is static, or proportional control action for stability and the second (involving $\chi_{k}(p)$ ) is integral action to enforce tracking of the desired limit profile $y_{\text {ref }}(p)$.

As a numerical example, consider the unstable along the pass special case of (1) given by

$$
\begin{aligned}
& A=\left[\begin{array}{cccc}
0.92 & 0.14 & -0.98 & 0.41 \\
-0.76 & -0.93 & -0.62 & 0.13 \\
0.68 & -0.65 & 1.02 & -0.81 \\
0.94 & 0.04 & 0.83 & 0.2
\end{array}\right], \quad E=\left[\begin{array}{c}
0.91 \\
0.59 \\
0.18 \\
0.31
\end{array}\right], \\
& B=\left[\begin{array}{ccc}
0.99 & -0.99 & 0.07 \\
0.07 & -0.94 & -0.63 \\
0.98 & -0.73 & 0.02 \\
-0.37 & 0.19 & -0.65
\end{array}\right], \quad B_{0}=\left[\begin{array}{cc}
-0.01 & -0.43 \\
0.29 & -0.13 \\
0.98 & 1.09 \\
1.09 & 0.17
\end{array}\right],
\end{aligned}
$$
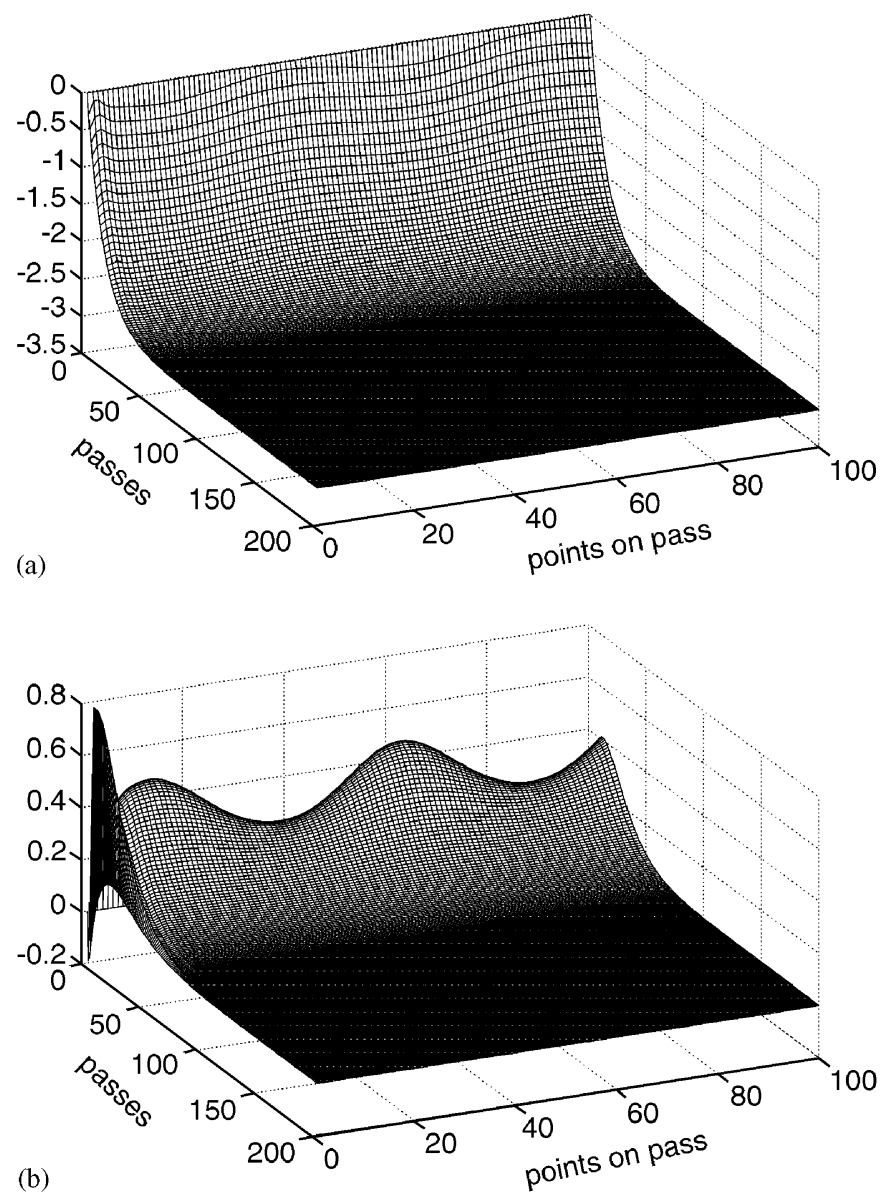

Fig. 1. (a) First channel response to $\left[\begin{array}{ll}-3 & 0\end{array}\right]^{\mathrm{T}}$; (b) Second channel response to $\left[\begin{array}{ll}-3 & 0\end{array}\right]^{\mathrm{T}}$.

$C=\left[\begin{array}{cccc}-0.75 & 0.75 & 0.31 & 0.84 \\ -0.86 & 0.99 & 0.33 & -0.84\end{array}\right], \quad F=\left[\begin{array}{c}-0.06 \\ 0.36\end{array}\right]$,

$D=\left[\begin{array}{ccc}-0.33 & -0.14 & 0.59 \\ -0.18 & 0.94 & -0.17\end{array}\right], \quad D_{0}=\left[\begin{array}{cc}1.11 & -0.66 \\ 0.46 & 1.23\end{array}\right]$

over the pass length $\alpha=100$. The disturbances $w(p)$ is two full periods of a sine wave. The control law matrices $K_{x}, K_{z 1}$ and $K_{z 2}$ resulting from applying Theorem 2 in this case are given.

In order to assess the quality of the controlled process performance we focus on the fact that $y_{\infty}$ is a $1 \mathrm{D}$ discrete linear system and follow the standard route of using a step signal applied in each of the two channels in turn. Figs. 1a and b show the controlled process response to the case when $y_{\text {ref }}(p)=$ $\left[\begin{array}{ll}-3 & 0\end{array}\right]^{\mathrm{T}}, 0 \leqslant p \leqslant 99$. Here the interaction in the second channel is initially quite large but the key feature is that the process converges relatively quickly to the limit profile which has exactly the along the pass dynamics predicted and, in particular, the integral term completely kills off the interaction. Figs. 2(a) and (b) show the controlled process responses in the case when $y_{\text {ref }}(p)=\left[\begin{array}{ll}0 & 3\end{array}\right]^{\mathrm{T}}, 0 \leqslant p \leqslant 99$, and the same general comments hold. (These simulations are for the boundary conditions $d_{k+1}=\left[\begin{array}{llll}-1.33 & -0.32 & 1.13 & -1.56\end{array}\right]^{\mathrm{T}}$ and zero initial 

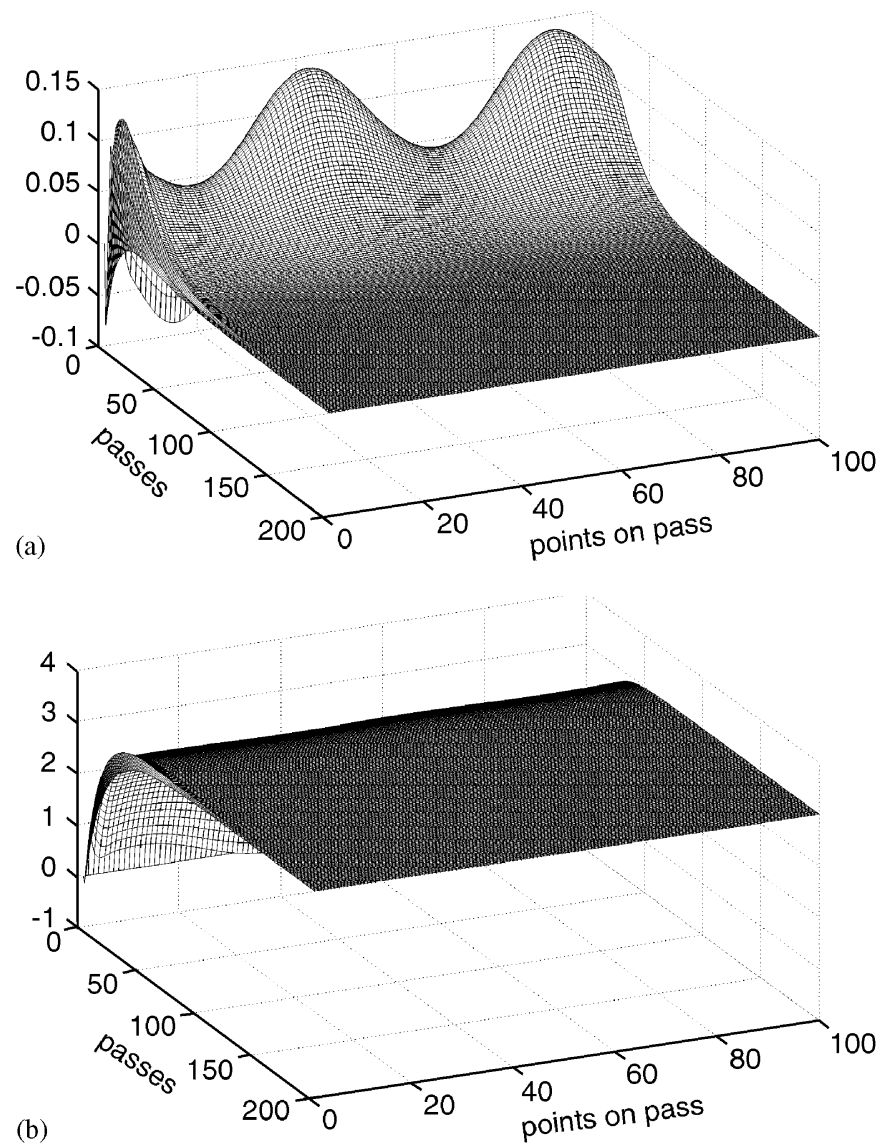

Fig. 2. (a) First channel response to $\left[\begin{array}{ll}0 & 3\end{array}\right]^{\mathrm{T}}$; (b) Second channel response to $\left[\begin{array}{ll}0 & 3\end{array}\right]^{\mathrm{T}}$.

pass profile $y_{0}(p)$.)

$K_{x}=\left[\begin{array}{cccc}-1.7945 & 1.0158 & 0.134 & 1.1219 \\ 0.5075 & -0.5836 & -0.5438 & 1.1854 \\ 0.3834 & -0.7341 & -0.6099 & -0.4623\end{array}\right]$, $\begin{aligned} K_{z 1}= & {\left[\begin{array}{cc}1.8756 & -0.6793 \\ 0.0952 & -1.3957 \\ -0.7203 & 0.3862\end{array}\right], } \\ K_{z 2}= & {\left[\begin{array}{cc}0.0673 & 0.0407 \\ 0.0441 & -0.0559 \\ -0.0606 & 0.0056\end{array}\right] . }\end{aligned}$

Further work could profitably directed towards tuning the converge rate. This has no immediate solution but one option may be to investigate the role of a Youla-Kucera parameterization. By ad-hoc numerical studies we conjecture that this design is not too conservative.

\section{Conclusions}

A control law design for discrete linear repetitive to achieve stability along the pass and a specified performance objective has been developed. This result only deals with disturbances which are constant from pass-to-pass and this reduces its general applicability. One alternative is to seek to attenuate the effects of such disturbances to a prescribed degree, e.g. in an $H_{\infty}$ or $H_{2} / H_{\infty}$ setting, which is an obvious area for further work.

\section{References}

Dullerud, G. E., \& D'Andrea, R. (2004). Distributed control of heterogeneous systems. IEEE Transactions on Automatic Control, 49(12), 2113-2128.

Gałkowski, K., Lam, J., Rogers, E., Sulikowski, B., Paszke, W., \& Owens, D. H. (2003). LMI based stability analysis and robust controller design for discrete linear repetitive processes. International Journal of Robust and Nonlinear Control, 13, 1195-1211.

Owens, D. H., Amann, N., Rogers, E., \& French, M. (2000). Analysis of linear iterative learning control schemes-a 2D systems/repetitive processes approach. Multidimensional Systems and Signal Processing, 11(1/2), 125-177.

Rogers, E., \& Owens, D. H., 1992. Stability analysis for linear repetitive processes, Lecture notes in control and information sciences series, Vol. 175, Berlin: Springer. 